eCommons@AKU

January 2017

\title{
Cultural practices of rearing preterm infants: A qualitative study in a tertiary care hospital, Karachi, Pakistan
}

\author{
Salima Sulaiman Gulamani \\ University of Toronto, Ontario, Canada \\ Ambreen Tharani \\ Aga Khan University, ambreen.tharani@aku.edu \\ Sharifa Bashir Lalani \\ Aga Khan University, sharifa.lalani@aku.edu \\ Kiran Shaikh \\ Aga Khan University, kiran.shaikh@aku.edu
}

Follow this and additional works at: https://ecommons.aku.edu/pakistan_fhs_son

Part of the Nursing Midwifery Commons

\section{Recommended Citation}

Gulamani, S. S., Tharani, A., Lalani, S., Shaikh, K. (2017). Cultural practices of rearing preterm infants: A qualitative study in a tertiary care hospital, Karachi, Pakistan. i-Manager's Journal on Nursing, 6(4), 6-13.

Available at: https://ecommons.aku.edu/pakistan_fhs_son/153 


\title{
RESEARCH PAPERS
}

\section{CULTURAL PRACTICES OF REARING PRETERM INFANTS: A QUALITATIVE STUDY IN A TERTIARY CARE HOSPITAL, KARACHI, PAKISTAN}

\author{
By \\ SALIMA SULAIMAN * \\ AMBREEN THARANI ** \\ SHARIFA LALANI *** \\ KIRAN SHAIKH **** \\ * Research Scholar, University of Toronto, Ontario, Canada. \\ $\star_{* *_{-} * \star * \star}$ Assistant Professor, Aga Khan University, School of Nursing and Midwifery, Karachi Pakistan. \\ Data Received: 19/11/2016 \\ Data Revised: 16/1/2017 \\ Data Accepted : 21/02/2017
}

ABSTRACT

The research work explores the cultural practices of feeding and hygiene of preterm infants in the Pakistani context. A Qualitative descriptive-exploratory design has been used. Purposive sampling was utilized to recruit 17 mothers, who met inclusion criteria from a largest government tertiary care hospital in Karachi, Pakistan. The semi-structured interview was used to collect the data, which lasted for approximately 45-60 minutes. Thematic analysis was conducted to analyze the key findings of the study. Welcoming newborn with 'honey' and herbal remedies were reported as a common ritual among mothers. Findings revealed differences in the time period of initial bath given to the preterm, ranges from 3 days to 1 month. These custom variations of rearing preterm have its own meaning in the prescribed culture; however, these customs are the insights to explore more on its effects on infants' health. This study provides useful insights into several practices of cultures, which serve as the determinants of infants' nutrition and health status. It provides an insight to the health care professionals to provide quality care to preterm infants.

Keywords: Feeding, Hygiene, Preterm, Qualitative.

\section{INTRODUCTION}

A cultural practice is an important aspect that needs to be considered by health care providers in order to deliver quality maternal and child health care services. Studies have shown the association between cultural practices, its belief and norms and family behaviors that influence attaining maternal and child health care (Ali \& HowdenChapman, 2007; Owoo \& Lambon-Quayefio, 2013). Importantly cultural practices would have an effect on the utilization of advanced health care practices which would not be utilized by families to make proper decisions. Many cultural norms are inherited in the family without knowing its significance or harmful effects on infants and mothers. For example, Taiwanese mothers are abide to follow several cultural rituals on their preterm infants imposed on them during the postnatal period (Lee, et al., 2009). Similarly, living with extended family has a greater influence on practicing cultural norms religiously. For instance, Latino mothers preferred to live in or near the home of their own parents to receive advice on preterm baby care from family members. Also, these mothers often seek help from family members in child care (Neu \& Robinson, 2008). Though the culture is considered as a significant contributor towards infants feeding and rearing practices, scarce researches are available to understand these diversifications. The most vulnerable from cultural practices are the preterm infants.

Preterm infants are the most vulnerable population. Preterm infant is defined as birth before completion of 37 weeks of gestation (World Health Organization, 2014). Each year around 15 million babies are born preterm and their survival chances vary dramatically around the world (Blencowe, et al., 2012). All resource regions: high, middle, and low report varying degrees of preterm birth rates (Beck, et al., 2009), ranging from $5 \%$ in developed countries to $25 \%$ in developing countries (Goldenberg, et al., 2008). The estimated global preterm birth is 9.6\% (Beck, et al., 2009). The rate of preterm birth in Pakistan is $15.7 \%$ (Lawn, et al., 2010). Preterm infants are at higher risk of adverse health outcomes than healthy, full term infants (Kair, et al., 
2015). Preterm infants are high risk of experiencing respiratory distress, hypoglycemia, temperature instability, and feeding difficulties than term infants (Engle, et al., 2007; Kramer, et al., 2000; Natalie, et al., 2014; Vohr, 2013). Therefore, cultural based practices and norms contribute significantly in the prevention and treatment of these associated health risks.

Breastfeeding has many health benefits for preterm infants like decreased infection and allergies (American Academy of Pediatrics, 2012). Preterm infants benefit more from breastfeeding than term infants. It is fundamental for infant's survival, growth, and development (Joseph, et al., 2013). WHO recommends exclusive breastfeeding of all infants until 6 months (WHO, 2014). In spite of all efforts, deployed as information, education, or training campaigns to promote mothers milk for the infants, the prevalence of exclusive breastfeeding is low (Li, et al., 2005). The probable reason could be that the psychosocial and cultural barriers exist to early breastfeeding (Garg, et al., 2010). According to (World Health Organization, 2014) non-exclusive breastfeeding includes prelacteal feeding is an important cause of infant morbidities. Also, there are other contributing factors for infection like improper and inadequate weaning foods and unhygienic feeding practices like bottle feeding.

Apart from feeding practices, there are certain other rearing practices which also influence the health of preterm infants and are widely prevalent among families and communities. These are oil massaging the baby, application of oil into eyes and ears, burping the baby, application of black carbon into the eyes, trimming of nails (Joseph, et al., 2013). Familiarity with these rearing practices is of considerable significance to a health worker serving the community. Specifically, in immigrant population, knowledge of cultural practices help health providers to provide efficient and accepted care to their client. (Steinman, et al., 2010) explored the feeding beliefs of Somali immigrants and identified the practice of discarding colostrum, as it is believed that it makes baby sick. Therefore, for promotion of infant health, the health care providers must identify the beneficial innocuous and harmful rearing practices. Also, it is important to explore the practices in preterm infant care before initiating any intervention plan. However, to date, no literature is available which appraises the cultural practices of rearing a preterm infant in Pakistani culture. Pakistan is a home country of several ethnic groups (e.g., Punjabi, Sindhi, Baloch, Pushton, etc.). This diverse cultural composition is depicted in every aspect of their lives and is most likely to vary in their preterm infant rearing practices. Thus, the purpose of the study was to explore the rearing practices of preterm infant in the Pakistani context. However, the present paper will describe the feeding and hygienic practices performed by Pakistani mothers that could impact on health status of preterm.

\section{Aims of the Study}

The aims of the study is to explore, a) the early parental experiences of mothers following the birth of preterm infant, b) the challenges of a mother in the early period following the birth of preterm infant, and c) the common cultural practices in the early period following the birth of the preterm infant.

\section{Objectives for the study}

- To explore the experiences of mothers in the early period following the birth of a preterm infant.

- To examine, the challenges of mothers in the early period following the birth of the preterm infant.

- To investigate, the common cultural practices of rearing a preterm infant in the early period.

\section{Research Methods}

\section{Study Design}

The study utilized the qualitative descriptive-exploratory design (Brink, 1998) to explore the early parental experiences, cultural practices, and challenges of mothers having a preterm infant.

\section{Study Setting}

The study was carried out in a largest government tertiary care hospital in Karachi, Pakistan. The hospital is located in the central hub of the city. The facility is utilized by the largest number of population and caters around two million patients annually, mostly from lower and lowermiddle population of patients. 


\section{Study Sampling}

Purposive sampling was utilized in the study to obtain cases deemed information-rich for the purpose of the study (Sandelowski, 2000). To receive meaning input from study participants, purposefully 10-15 mothers who delivered preterm birth at the hospital, and were attending the pediatric outpatient clinic for follow up with a baby between two to three months was included (Polit, et al., 2001). Mothers who were able to speak and understand Urdu (national language) and/or Sindhi (provincial language) were recruited. However, the data was collected from 17 mothers who were meeting the above mentioned inclusion criteria until data saturation was reached.

\section{Ethical Consideration}

The study was approved by the Aga Khan University Hospital's Ethics Research Committee (ERC). Permission was taken from the Medical Superintendent (MS) of the data collection site. The mothers fulfilling the eligibility criteria were recruited. Participant's confidentiality and anonymity were maintained throughout the study. Anonymity was ensured by coding participants' identity while handling the data (Polit and Beck, 2010). All manual data and tapes were kept in lock and key in a secure place with research team. Electronic voice recorded interviews and data in soft copy were password protected and accessible only by the researchers.

\section{Data Collection}

Informed consent in Urdu/Sindhi was obtained from the mothers. Prior to the interview, a demographic data form was filled by the researchers to have baseline information of the participants. Interviews were conducted with the help of a structured interview guide (Polit and Beck, 2010). The interview guide was piloted on two mothers to ensure validity and reliability of the tool. The interview guide was modified based on the feedback received on pilot testing. Each interview lasted for approximately 45-60 minutes.

\section{Data Analysis}

(Morse, 1994), qualitative data analysis processes were applied within the descriptive-exploratory design. The interview guide consist of three major focus areas consisting,
- Experiences of mothers.

- Challenges.

- Common cultural practices in the early period following the birth of preterm infant.

The data analysis was proceeded by,

- Transcribed interview of the participants coded for analyzing data.

- Identified emerging themes in the data.

- Labeled the themes with significant code words.

- Codes were clustered and labeled using broader themes and sub themes throughout analytic process.

- The thematic strands were weaved together into an integrated picture of phenomena under investigation. The qualitative content analysis was used as analytic method.

\section{Rigor and Trustworthiness}

(Lincoln and Guba, 1985) suggested four criteria for ensuring the trustworthiness of a qualitative research.

- Credibility or internal validity.

- Dependability, reliability, or stability,

- Confirmability or objectivity.

- Transferability, external validity, or generalizability.

In this study, credibility was also ensured by covering the data through categories (Graneheim \& Lundman, 2004). Member check was done following the interviews, to correct the errors of interpretation. Dependability can be met through the obtaining credibility of the findings. Conformability was ensured by confirming an audit trail. Therefore, the record of raw data (interview transcript) and the data reduction notes (theoretical notes) was kept by the researcher. Transferability was ensured by documenting a thick description of the findings could be transferable or applicable in other settings (Guba \& Lincoln, 1989).

\section{Findings}

The study participants encompassed 17 mothers aged between $20-38$ years. Nearly $82 \%(n=14)$ of the mothers were housewives and remaining were either self-employed or labors. In terms of educational status, almost $52 \%(n=9)$ of the mothers were illiterates, whereas the rest of the 
mothers (48\%) had primary $(n=3)$ or secondary $(n=5)$ level of education. All the mothers were permanent resident of Pakistan, representing various cultures, including Sindhi, Punjabi, Kaachi, Balochi, Mahajir, Pathan, and Bengali. However, able to understand and speak Urdu and/or Sindhi. The family income of $65 \%(n=11)$ of mothers participated in this study ranged PKR 5000-20000. Whereas, five mothers $(45 \%)$ were not ready to reveal their family monthly income. Unexpectedly, one of the participants informed family income less than PKR 5000.

The study unveiled various cultural, feeding, and hygiene practices being performed by mothers to foster health and well-being of their preterm babies. In addition, rich data were obtained regarding fears and stresses encountered by mothers in rearing their preterm. Also, the role of the family was identified as instrumental by mothers to manage their preterm. As mentioned earlier, the current paper will elaborate the findings of feeding and hygiene practices undertaken by mothers of preterm in Pakistani context.

\section{Feeding Practices}

Findings suggested cultural dimensions of various feeding practices of mothers of preterm babies. Welcoming a newborn with 'honey' is reported as a common practice among mothers in Pakistan. They strongly felt that this is a traditional practice that is necessary to be done. They also affirmed that the baby will shadow the person who gives honey to him/her during their welcome ritual. As one of the participants stated:

"In our culture we believe that) child will shadow person who will first feed honey to him. We give diluted form of honey with water until the child turns three months" (p7).

One of the mothers only gave honey water for three months and did not breastfeed her baby. Absence of breastfeeding can lower the immunity and develop illnesses in an infant, She said,

"I was giving him honey for consecutive 3 days and did not feed him milk and till 3 months I was giving honey and water" (p9).

However, few of the participating mothers reported opposing the cultural norm of feeding honey as to them it may have a negative impact on baby as being preterm. As a mother stated:

"I don't want him to become ill (as he is already weak)... so I didn't give him honey or anything else besides breastfeed" (p16).

In the same line, few of the participants shared feeding with herbal tea and water is also practiced, instead of honey, to babies at the time of birth. Some of the mothers still practice feeding these herbal remedies frequently to maintain babies' digestion. These herbs have been usually homemade like 'wavrine', 'wakomba', and 'ghutki' (locally available herbs). Whereas, some of the mothers were using the available form of prepared herbs like 'naunehal' (an herbal water prepared by a pharmaceutical company).

"She had constipation. So I gave her 'Jiwanghutki' (herbal remedy) daily till she is 2 months old. I still give it to her when needed to ensure her digestion and prevent from colic" (p14).

Almost $90 \%$ of the mothers $(n=15)$ were breastfeeding their babies along with top feed, cow's milk and weaning diet. Mothers reported their struggle in feeding preterm babies as to them, they were weak, as a mother shared:

"Every time when I gave her milk, her breathing gets blocked. Then I put her on my shoulder and give a small portion of milk slowly. I continuously rub her back so that the milk moves down in her stomach and she does not vomit it out"(p1).

The practices of weaning were reported as early as at the age of one month. However, some of the mothers were strictly following the doctor's advice on the cost of their cultural practices. The common weaning diet included flour, dalya (grinded wheat), semolina (sooji), sajo (sabudana), prepared powdered cereal (wheat based cerelac), and biscuits. While explaining the method of preparing diet, a mother stated:

"First we make flour red and then add water, milk and sugar in it. We cook for a little while and then feed to a child once cold"' (p9).

Interestingly, one of the mothers reported that she administered juice to baby to treat diarrhea, as she reported: 
"He had diarrhea since 10-15 days, so I gave him orange juice. Now whenever he gets diarrhea, I feed him with tetra packjuice" (p3).

\section{Hygiene Practices}

The majority of the mothers reported that they haven't given bath to the newborn after birth. The first bathing time varies from 3 days to 1 month and mothers were able to rationalize it with the fragility of the newborn. Therefore, they commonly clean the newborn with cloth soaked in water and lotion, till they gave first bath. Bathing babies with delicacy is reported by mother as one of the mothers narrated her experience as:

"First I warm the water. Then hold the baby in my hand and apply soap slowly. After that, I pour little bit amount of water and prevent his nose and ear from water. Then I turned him back and give a bath" (p2).

The variation in practices of using soap was also noted. As many of the mothers were using locally available antiseptic soaps 'Dettol' and 'Safeguard' to bath their newborns. As one of the mothers stated:

"On second day of his birth, I gave him bath with Dettol soap so he is clean and free from all infections" (p6).

Interestingly, most of the mothers reported bathing newborn on their legs as they feel it will prevent them from falling. As one of the mothers narrated:

"I put the baby on my legs and then give bath. In this way my baby will feel secure and will not cry because she can see me. I don't pour water on her face; I just put on her head...." (p1 1).

As part of bathing ritual, mothers usually massage babies body with lotion and various oils, including mustard, rose, fish, and coconut oil before bath. The prime purpose of massaging infant with oil is to give muscle strength. As a mother narrated:

"I use mustard oil and rose oil to massage my baby. I also use fish oil so that baby can get strength and can easily hold his neck and stand on his foot soon" (p10).

Less than one third of the mothers reported rare practice of cleaning newborn's umbilical cord with coconut oil and consider that it fosters healing.

"I applied coconut oil on his cord and cleaned with cotton.
I do it daily so that his cord stays clean and prevent from infection" (p17).

Following the bath, a common practice of grooming newborn was reported by application of black carbon (kajal) in their eyes. The purpose of the application of kohl was to improve their eyesight, prevent from bad evil, and beautification of the newborn.

"I groom my child by applying kajal after bath. I gave him first bath on fifth day after his birth and applied kajal too. My mother in law always says that it will prevent child from evil eye and child will never get sick" (p1).

Mothers often prepare khol at home, as one of the mothers shared the steps of preparing khol as:

"We first put coconut oil in a spoon, then wrap a piece of cloth and put sesames oil in it. Than prepare khol (kajal) from it which is chemical free and safe for baby. Then we apply itto baby daily" (p1).

\section{Discussion}

Rearing preterm infants form an important aspect of community practices. Cultural health beliefs and practices are still strongly practice, especially among developing countries. Health care workers need to be sensitive with cultural practices among the population they served. Some may be harmful and some may be beneficial for preterm infants. Different cultural practices related to feeding and hygiene were highlighted in this study. Study findings suggest that majority mothers were breastfeeding infants which is consistent with others studies (Steinman, et al., 2010; Mohammed, 2014). Also, the study highlights that infants were provided with top feed which include cow milk, weaning, guthi, etc. The findings are consistent with the literature that has found that infants were breastfeed (Joseph, et al., 2013; Memon, et al., 2006) along with a combination of animal milk, formula milk, semi-solids, solid diet (Joseph, et al., 2013), butter, ghutti, tea (Memon, et al., 2006), glucose water (Sreedevi \& Rao, 2015). Findings indicate usage of prelacteal food with its belief rooted in the culture. For example, usage of honey in infancy have been seen in the study. Similar findings have been reported in a study that has found practice of pre-lacteal feeding of honey, water, butter, ghutti, and tea, due to various reasons such as maternal illness, pain at operative sites, 


\section{RESEARCH PAPERS}

generalized weakness, and perception that milk will come in 2-3 days, were the major reasons for delay in breastfeeding for 2 to 3 days as well, whereas honey was preferred to be given as it is considered hot (Memon, et al., 2006); while the purpose of these food supplements in the present study was different. Honey was used as it is believed that the person introducing honey in the baby's mouth is transferring his/her qualities to the baby. Herbal tea and juice was used to improve digestion and treat diarrhea, respectively. Present study reported that weaning was started at one month of age, which is consistent with the findings which also reported that supplementary milk was started at 2-4 weeks or earlier than 6 months of the infant's age (Memom, et al., 2006; Mohammed, 2014).

Hygiene practices were another important area where mothers found to face many variations in practices and were challenging. The study findings, highlight differences in the time period of baby bath given to the preterm ranging from 3 days to 1 month. The findings are consistent with the study, which also reported delays initiation of bath after 1 week (Joseph, et al., 2013). Whereas, bathing occurred soon after delivery in Nigerian, but was delayed for several hours or until the next day for most Tanzanian and Ethiopian narrative mothers (Adejuyigbe, et al., 2015). Present study also showed the ritual of massage which is consistent with finding of the study which also emphasized the practice of oil massage before bath by mothers (Joseph, et al., 2013). It also suggested application of carbon (kajal) in preterm eye where the literature highlights the practice of oil application to the eyes or ears of infants (Joseph, et al., 2013). Interestingly, the finding indicates application of coconut oil at umbilical for cleaning, in contrast, whereas, Sutan and Berkat (2014) reported that the majority of the participants didn't apply traditional ingredient or alcohol for umbilical cord care.

\section{Limitations}

This study provides useful insights into several practices of cultures which serve as the determinants of newborn nutrition and health status, therefore understanding these rituals are important especially when they are preterm. However, there were some limitations of the study. The study was conducted in a government hospital where people from various places visited the facility and mothers were speaking many different languages, however, mothers who spoken Urdu and Sindhi were recruited due to language barrier.

\section{Recommendations}

This paper examines the feeding and hygiene practices of preterm infants, further exploration of other cultural practices is needed. Also, the data were primarily collected in the hospital setting, further investigation is therefore needed in exploring the rearing practices of preterm infants in a community setting. Due to the limited research on preterm infants, a qualitative study that investigates the effects of cultural practices on preterm infants seem quite logical. A final recommendation includes a comparison study between the cultural practices of term and preterm infants to identify the differences in the rearing practices.

\section{Conclusion}

Rearing practices of preterm infants described many cultural rituals which focus on feeding and hygiene practices of infants. These customs have its own meaning in the prescribed culture that provides an insight for the health care professionals; therefore, an exploration of cultural practices is important before the initiation of any intervention.

\section{Conflict of Interest}

The authors declare that that there is no conflict of interest in regards to the research, authorship, and publication of this paper.

\section{References}

[1]. Adejuyigbe EA, Bee MH, Amare Y, Omotara BA, Iganus RB, Manzi F et al. (2015). "Why not bathe the baby today? A qualitative study of thermal care beliefs and practices in four African sites". BMC Pediatrics, Vol. 15, pp. 156-162.

[2]. Ali A and Howden-Chapman P, (2007). "Maternity Services and the Role of the Traditional Birth Attendant, Bidan Kampong, in Rural Malaysia". Journal of Public Health ManagementPractice, Vol. 13, No.3, pp.278-286.

[3]. American Academy of Pediatrics, (2012). "Policy statement breastfeeding and the use of human milk". Pediatrics, Vol. 129, No.3, pp.e827-e844. 


\section{RESEARCH PAPERS}

[4]. Beck S, Wojyla D, Say L, Betran AP, Merialdi M, Requejo $\mathrm{JH}$, ef al., (2009). "The worldwide incidence of preterm birth: A WHO systemic review of maternal mortality and morbidity". The Bulletin of World Health Organization, Vol.88, pp.31-38.

[5]. Blencowe $H$, Cousens $S$, Oestergaard M, Chou D, Moller AB, et al., (2012). "National, Regional and Worldwide Estimates of Preterm Birth". The Lancet, Vol.379, No.9832, pp.2162-72.

[6]. Brink PM, (1998). "Exploratory Designs". In: Brink PJ and Wood MJ (Eds.), Advanced Design in Nursing Research. Thousand Oaks: Sage, pp.308-333.

[7]. Engle WA, Tomashek KM, and Wallman C, (2007). "Late Preterm Infants: A Population at Risk". Pediatrics, Vol.120, pp. 1390-1401.

[8]. Garg R, Deepti S, Padda A, and Singh T, (2010). "Breastfeeding knowledge and Practices among Rural Women of Punjab, India: A Community-Based Study". Breastfeed Medicine, Vol.5, No.6, pp.303-307.

[9]. Goldenberg RL, Culhane JF, lams JD, and Romero R, (2008). "Epidemiology and causes of preterm birth". The Lancet, Vol.37, No.9606, pp.75-84.

[10]. Graneheim, U. H., \& Lundman, B. (2004). "Qualitative content analysis in nursing research: Concepts, procedures, and measures to achieve trustworthiness". Nurse Education Today, Vol.24, pp. 105-1 12.

[1 1 ]. Guba, E. G., \& Lincoln, Y. S. (1989). Fourth Generation Evaluation. Newbury Park, CA: Sage.

[12]. Joseph N, Unnikrishnan B, Naik VA, Mahantshetti NS, Mallapur MD, Kotian SM, and Nelliyanil M, (2013). "Infant Rearing Practices in South India: A Longitudinal Study". Journal of Family Medicine of Primary Care, Vol.2, No. 1, pp.37-43.

[13]. Kair LR, Flaherman VJ, Newby KA, and Colaizy TT, (2015). "The experience of breastfeeding the late preterm infant: Qualitative study". Breastfeeding Medicine, Vol.10, No.2, pp. 102-106.

[14]. Kramer MS, Nemissie K, Yang H, et al., (2000). "The Contribution of Mild and Moderate Preterm Bitth to Infant Mortality. Fetal and infant health study group of the Canadian perinatal surveillance system". JAMA, Vol.284,
No.7, pp.843-849.

[15]. Lawn JE, Gravett MG, Nunes TN, Rubens C, and Stanton C, (2010). "Global report on preterm birth and still birth (1 of 7): Definitions, description of the burden, and opportunities to improve data". BioMed Central Pregnancy and Childbirth, pp. 1471-2393.

[16]. Lee SC, Long A, and Boore J, (2009). "Taiwanese women's experiences of becoming a mother to a very low birth weight preterm: A grounded theory study". International Journal of Nursing Studies, Vol.46, pp.326336.

[17]. Li R, Darling N, Maurice E, Barker L, and GrummerStrawn LM. (2005). "Breastfeeding Rates in the United States by Characteristics of the Child, Mother, or Family: The 2002 National Immunization Survey". Pediatrics, Vol.115, No.1, pp.31-37.

[18]. Lincoln,Y. S., \& Guba, E. G. (1985). Naturalistic Inquiry. Newbury Park, CA: Sage.

[19]. Memon Y, Sheikh S, Memon A, and Memon N, (2006). "Feeding beliefs and practices of mothers/ caregivers for their infants". Journal of Liaquat University of Medical Health Sciences, Vol. 1, pp. 8-13.

[20]. Mohammed SJS, (2014). "Infants feeding and weaning practices among mothers in Northern Kordofan state, Sudan". European Scientific Journal, Vol. 10, No.24, pp. 165-181.

[21]. Morse JM, (1994). "Emerging from the data: The cognitive processes of analysis in qualitative inquiry". In: Morse JM (Ed.), Critical issues in Qualitative Research Methods. Canada: Sage. pp.22-43.

[22]. Natalie M, Ventura M, Colombo M, et al., (2014). "Short-term respiratory outcomes in late preterm infants". Italian Journal of Pediatric, Vol.40, No. 1, pp.52-62.

[23]. Neu M and Robinson J, (2008). "Early Weeks after Premature Birth as Experienced by Latina Adolescent Mother". Mother and Child Nursing, Vol.33, No.3, pp.32-38.

[24]. Owoo N and Lambon-Quayefio MP, (2013). "National health insurance, and social influence and antenatal care use in Ghana". Health Economics Review, Vol.3, No.19, pp. 1-12.

[25]. Polit DF, Beck CT, and Hungler BP, (2001). Essential of 


\section{RESEARCH PAPERS}

Nursing Research: Methods, Appraisal and Utilization, $5^{\text {th }}$ ed. Philadelphia: Lippincott.

[26]. Polit DF and Beck CT, (2010). Nursing Research: Generating and Assessing Evidence for Nursing Practice. Philadelphia: Lippincott.

[27]. Sandelowski M, (2000). "Whatever happened to qualitative description?" Research in Nursing and Health, Vol.23, pp.334-340

[28]. Sreedevi, B. \& Rao, R. N. (2015). "A Longitudinal Study on Feeding Practices and Morbidity Patterns of Infants in a Rural Field Practice Area of Thadikonda, Guntur, AP". Journal of Nursing and Health Sciences, Vol.4, No. 1, pp.3639.

[29]. Steinman L, Doescher M, Keppel GA, et al., (2010). "Understanding infant feeding beliefs, practices, and preferred nutrition education and health provider approached: An exploratory study with Somali mothers in USA". Maternal Child Nutrition, Vol.6, No. 1, pp. 1-16.

[30]. Sutan, R. \& Berkat, S. (2014). "Does cultural practice affects neonatal survival- a case control study among low birth weight babies in Aceh Province, Indonesia". BMC Pregnancy and Childbirth, Vol. 14, No.342, pp. 1-13.

[31]. Vohr, B. (2013). "Long-term outcomes of moderately preterm, late preterm, and early term infants". Clinical Perinatal, Vol.40, pp.739-751.

[32]. World Health Organization (2014). WHA Global Nutrition Targets 2025: Breastfeeding Policy Brief. Retrieved from http://www.who.int/nutrition/topics/globaltargets_breastfee ding_policybrief.pdf?ua $=1 \& \mathrm{ua}=$

\section{ABOUT THE AUTHORS}

Salima Sulaiman is currently receiving her PhD from University of Toronto, Ontario, Canada. She completed her Master's of Science in Nursing from the Aga Khan University School of Nursing and Midwifery, Karachi, Pakistan. Her area of interest is to understand the Mental Health of Pregnant Women and Postpartum Women Caring for Infants.

Ambreen Tharani is currently working as an Assistant Professor at Aga Khan University School of Nursing and Midwifery, Karachi, Pakistan. She completed her BSc Nursing from the Aga Khan University and Masters in Education, Health Promotion and International Development from the IOE, University College of London. Her research interest focuses on Mental Health Promotion and Educational Challenges.

Sharifa Lalani is currently working as an Assistant Professor at Aga Khan University School of Nursing and Midwifery, Karachi, Pakistan. She received her Master's of Science in Nursing from the Aga Khan University School of Nursing and Midwifery. Her area of interest is Maternal and Child Health. Her recent work identifies the social determinants that affects Mental Health of Pregnant Women and Develop Strategies that consider the Needs of Pregnant Women.

Kiran Shaikh is currently working as an AssistantProfessor at Aga Khan University School of Nursing and Midwifery, Karachi, Pakistan. She received her Master's of Science in Nursing from the Aga Khan University School of Nursing and Midwifery. Her area of interest is Maternal and Child Health. Her recent research work is on the Psychosocial Health of Pregnant Women in Karachi.
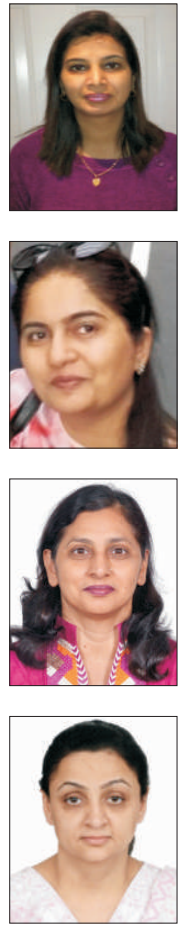